\title{
Prism-Coupling of Light into Narrow Planar Optical Waveguides
}

\author{
W. A. PASMOOIJ, P. A. MANDERSLOOT AND M. K. SMIT
}

\begin{abstract}
The efficiency and the selectivity of the prism-coupler for exciting single modes in narrow planar optical waveguides is investigated both theoretically and experimentally. Some practical rules are given for obtaining selective and stable excitation.
\end{abstract}

\section{LIST OF SYMBOLS}

$b_{y 0} \quad$ Distance over which the beam widens from $w_{y 0}$ to $w_{y 0} \sqrt{2}$.

$b_{\chi 0} \quad$ Distance over which the beam widens from $w_{x 0}$ to $w_{x 0} \sqrt{2}$.

$G_{l}(\chi, \zeta)$ Lateral input beam shape.

$G_{t}(\xi, \zeta)$ Transverse input beam shape.

$G_{y}(y, z)$ Input beam shape in the $y-z$ plane.

$G_{z}(z) \quad$ Input beam shape along the $z$-axis.

$k_{0} \quad$ Wavenumber in vacuum.

$l_{c} \quad$ Coupling length.

$n_{p} \quad$ Prism refractive index.

$R_{y}(z) \quad$ Radius of the input-beam lateral phase fronts in the $y-z$ plane.

$R_{\chi}(\zeta) \quad$ Radius of the input-beam lateral phase fronts.

$u, v \quad$ Normalized transverse propagation constants.

$U_{e}(y, z)$ Required input beam shape in the $y-z$ plane, matching the lateral mode profile and the longitudinal exponential profile.

$U_{i}(y, z)$ Applied input beam.

$U_{m}(y) \quad$ Lateral mode profile.

$V \quad V$ parameter or normalized film parameter.

$w_{g} \quad$ Waveguide lateral width.

$w_{m} \quad$ Mode profile $1 / e$ half width.

$w_{y}(z) \quad$ Lateral $1 / e$ half width of the input beam in the $y-z$ plane.

$w_{y 0} \quad$ Minimum of $w_{y}(z)$.

$w_{z} \quad 1 / e$ half width of Gaussian input beam measured along the $z$-axis.

$w_{\eta} \quad 1 / e$ half width of the angular coupling efficiency curve.

$w_{\xi} \quad 1 / e$ half width of $G_{t}$.

Manuscript received August 4, 1987; revised February 8, 1988.

W. A. Pasmooij and P. A. Mandersloot are with the Dr. Neher Laboratory (PTT), Leidschendam, The Netherlands.

M. K. Smit is with Delft University of Technology, Faculty of Electrical Engineering, Laboratory of Telecommunications and Remote Sensing Technology, 2600 GA Delft, The Netherlands.

IEEE Log Number 8822062

\author{
$w_{\chi}(\zeta) \quad 1 / e$ half width of $G_{l}$. \\ $w_{x 0} \quad$ Minimum of $w_{\chi}(\zeta)$. \\ $x, y, z \quad$ Cartesian coordinate system with the bot- \\ tom of the prism as the $y-z$ plane. \\ $z_{c} \quad$ Optimal $z$-position of the prism corner rel- \\ ative to the input-beam center $(z=0)$. \\ $\beta \quad$ Mode propagation constant. \\ $\Delta \theta \quad$ Deviation from phase-match angle. \\ $\eta \quad$ Coupling efficiency. \\ $\theta \quad$ Input beam incidence angle. \\ $\theta_{0} \quad$ Phase-match angle. \\ $\xi, \chi, \zeta \quad$ Cartesian coordinate system with the optical \\ axis of the input beam as $\zeta$-axis.
}

\section{INTRODUCTION}

$\mathrm{T}$ THE METHODS most commonly used for coupling power into or out of a planar optical circuit are prismcoupling and butt-coupling. Prism-coupling was introduced for this purpose by Ulrich and Tien [1], and described in more detail by the same authors in later publications [2], [3]. A compact description has been given by Unger [4].

Prism-coupling differs from butt-coupling in that it is mode selective. This is well known for the case of transverse film modes, and much less so for the case of lateral waveguide modes. Excitation of lateral modes was reported by Millar et al. [5] and Simova et al. [6], but without quantitative details with respect to the selectivity. A theoretical analysis of the coupling mechanism, including the excitation of lateral modes, was given by Wang and Laybourn [7]. They calculated the coupling efficiency between a plane input wave with finite cross section and a number of waveguide modes, as a function of both the incidence angle and the skew angle.

In the present article the analysis will be extended to include the effects of diffraction, which will be shown to play a dominant role for small focal widths of the input beam. The theoretical predictions are compared with experimental results. Furthermore a number of mechanisms reducing coupling selectivity and stability will be discussed, and some practical rules will be given for obtaining good stability and selectivity.

\section{Experimental Coupling Configuration}

The experiments presently reported were conducted at $633 \mathrm{~nm}(\mathrm{He}-\mathrm{Ne})$ wavelength on an embedded ridge-type 


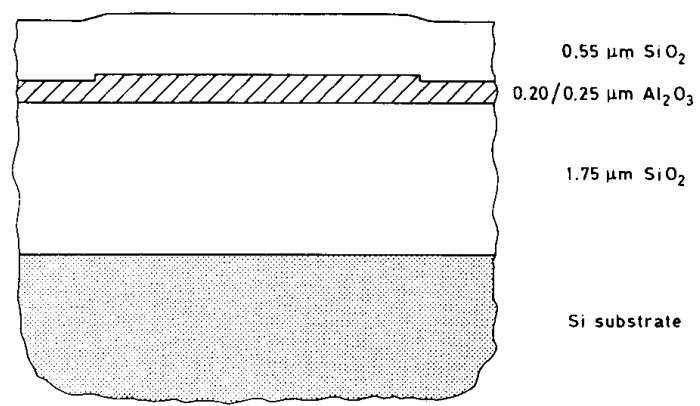

Fig. 1. Cross section of an embedded ridge-type waveguide structure.

waveguide structure, consisting of a RF-diode sputtered $\mathrm{Al}_{2} \mathrm{O}_{3}$ film ( $n \simeq 1.69$ ) on a thermally oxidized silicon substrate $\left(n=1.46\right.$ ), as depicted in Fig. 1. The $\mathrm{SiO}_{2}$ film thickness was $1.75 \mu \mathrm{m}$, which proved to be sufficient for isolating the guided wave from the absorbing silicon substrate. The lateral waveguide structure was formed in the $\mathrm{Al}_{2} \mathrm{O}_{3}$ film by atom-beam milling. After milling we covered the whole structure with a RF-magnetron sputtered $\mathrm{SiO}_{2}$-layer ( $n \simeq 1.46$ ) which was simultaneously used as a gap layer. The coupling length of the prism-coupling is precisely controlled by the thickness of this layer. To avoid the occurrence of an additional air gap between the prism and the top layer we applied a contacting liquid with the same refractive index as the prism $\left(\mathrm{CH}_{2} \mathrm{I}_{2}, n \simeq 1.74\right)$. The coupling prisms were coated with chrome on opposite faces to avoid leakage of scattered light directly from the input to the output prism. More details are provided in [8] and [9].

The silicon-based $\mathrm{SiO}_{2} / \mathrm{Al}_{2} \mathrm{O}_{3} / \mathrm{SiO}_{2}$ waveguide system is interesting for hybrid applications in conjunction with III-V semiconductor lasers. A mounting method for buttcoupling semiconductor lasers to a silicon-based circuit is presently being developed at the authors laboratory.

The coupling approach, described above, seems to be applicable also for quaternary semiconductor circuits, using a silicon prism and a high-index low-melting-point eutectic as contacting "liquid".

\section{The Three-Dimensional Coupling Mechanism}

\section{A. Coupling Efficiency}

Coupling efficiency for the three-dimensional coupling situation can be inferred in much the same way as for the two-dimensional case by applying the overlap-integral theorem in two dimensions:

$$
\eta=\frac{\left|\iint U_{i}(y, z) U_{e}^{*}(y, z) d y d z\right|^{2}}{\iint\left|U_{i}(y, z)\right|^{2} d y d z \iint\left|U_{e}(y, z)\right|^{2} d y d z} .
$$

In this formula $U_{i}(y, z)$ describes the $y-z$ dependence of the input beam at the bottom of the prism (see Fig. 2). $U_{e}(y, z)$ represents the exponential input field required for 100-percent coupling efficiency (i.e., the reciprocal of

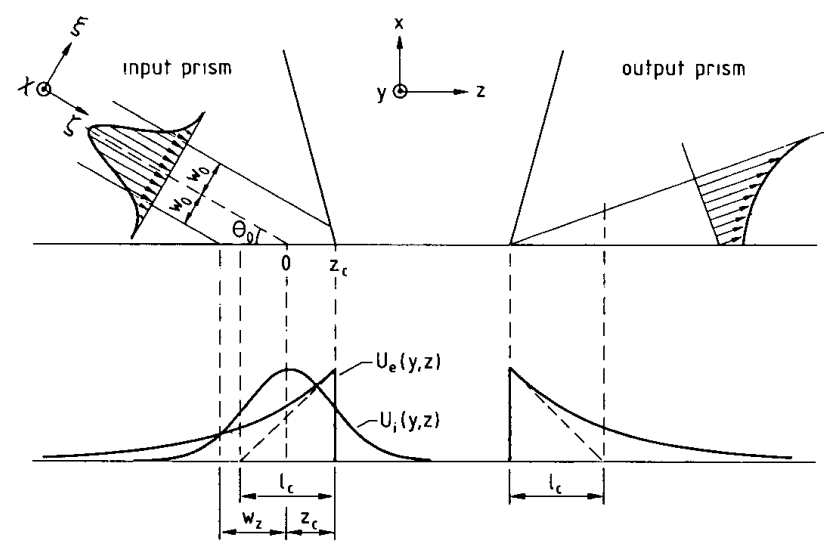

Fig. 2. Transverse coupling geometry and coupling parameters.

the field coupled out of the waveguide). In the three dimensional situation $\eta$ is dependent on the incidence angle as well as on the skew angle of the input beam. In the following analysis the skew angle will be supposed to be zero, being the optimal situation for exciting the fundamental mode. For exciting odd modes a (small) skew angle is essential; experiments show that the effects on the selectivity are small, however.

We will first compute $\eta$ at the phase match angle (i.e., $\theta_{0}=\arccos \left(\beta / n_{p} k_{0}\right)$, in which $\beta$ is the mode propagation constant, $n_{p}$ the prism index, and $k_{0}$ the vacuum wavenumber), yielding information about the maximum coupling efficiency for a focused beam. To begin, approximate expressions for $U_{i}(y, z)$ and $U_{e}(y, z)$ will be derived.

We assume the input beam to be Gaussian both in the transverse and lateral direction, but with different beamparameters due to lateral focusing (with a cylindrical lens):

$$
U_{i}(\xi, \chi, \zeta)=G_{t}(\xi, \zeta) G_{l}(\chi, \zeta) e^{-j n_{p} k_{0} \zeta}
$$

where $\xi, \chi$ and $\zeta$ are the transverse, the lateral, and the longitudinal coordinates in a Cartesian coordinate system in which the $\zeta$-axis coincides with the beam axis and:

$$
\begin{aligned}
G_{t}(\xi, \zeta)= & e^{-\xi^{2} / w_{\xi}^{2}} \\
G_{l}(\chi, \zeta)= & {\left[\frac{w_{\chi 0}}{w_{\chi}(\zeta)}\right]^{1 / 2} \exp \left[-\left\{\chi^{2} / w_{\chi}^{2}(\zeta)\right\}\right.} \\
& \left.-j \frac{n_{p} k_{0} \chi^{2}}{2 R_{\chi}(\zeta)}+\frac{1}{2} j \arctan \left(\frac{\zeta}{b_{\chi 0}}\right)\right] \\
w_{\chi}(\zeta)= & w_{\chi 0}\left\{1+\left(\frac{\zeta}{b_{\chi 0}}\right)^{2}\right\}^{1 / 2} \\
R_{\chi}(\zeta)= & \zeta \cdot\left\{1+\left(\frac{b_{\chi 0}}{\zeta}\right)^{2}\right\} \\
b_{\chi 0}= & n_{p} k_{0} w_{\chi 0}^{2} / 2 .
\end{aligned}
$$


Our notation follows that of [10]. For the transverse beam shape (equation (3a)), $w_{\xi}$ is such that its $\zeta$-dependence is negligible in the coupling region. In the formula for the lateral beam shape description (equation (3b)) the term between braces accounts for the amplitude reduction due to the broadening of the beam. In the exponent the first term describes the Gaussian beam profile, and the second term the spherical phase fronts. The third term disappears at $\xi=0$ and approaches to $\pm j(\pi / 4)$ for large values of $\zeta$.

For the transverse coupling problem the optimum coupling occurs if the beam focus coincides with the intersection point with the bottom of the prism. We will assume that the same holds for the lateral focus. Although beam shaping in two dimensions might shift the optimal focal positions, it is believed that the effects on the following analysis will be small.

Using (3a)-(3e), the following expression for $U_{i}(y, z)$ in the $y-z$ plane (i.e., at the bottom of the prism) has been derived:

$$
U_{i}(y, z)=G_{z}(z) \cdot G_{y}(y, z) \cdot e^{-j n_{p} k_{0} z \cos \theta}
$$

where

$$
\begin{aligned}
G_{z}(z)= & e^{-z^{2} / w_{z}^{2}} \\
G_{y}(y, z)= & \left\{w_{y 0} / w_{y}(z)\right\}^{1 / 2} \cdot \exp \left[-y^{2} / w_{y}^{2}(z)\right. \\
& \left.-j \frac{n_{p} k_{0} y^{2}}{2 R_{y}(z) \cos \theta}+\frac{1}{2} j \cdot \arctan \left(\frac{z}{b_{y 0}}\right)\right] \\
w_{z}= & w_{\xi} / \sin \theta \\
w_{y}(z)= & w_{y 0}\left\{1+\left(\frac{z}{b_{y 0}}\right)^{2}\right\}^{1 / 2} \\
R_{y}(z)= & z\left\{1+\left(\frac{b_{y 0}}{z}\right)^{2}\right\} \\
w_{y 0}= & w_{x 0} \\
b_{y 0}= & \frac{1}{2} \frac{n_{p} k_{0}}{\cos \theta} w_{y 0}^{2} .
\end{aligned}
$$

For the required exponential field $U_{e}(y, z)$ (see Fig. 2) the $y$-dependence is described by the fundamental mode profile $U_{m}(y)$. To simplify the expressions we will approximate this mode profile as a Gaussian profile $\exp \left(-y^{2} / w_{m}^{2}\right)$ in which $w_{m}$ is chosen such that the effective widths $\left(U_{m}(y)\right.$ having unit peak amplitude) are equal:

$$
\int_{-\infty}^{+\infty} U_{m}^{2}(y) d y=\int_{-\infty}^{+\infty} e^{-2 y^{2} / w_{m}^{2}} d y
$$

The effect of this approximation on the results computed according to (1) will be small, the overlap of $U_{m}(y)$ and its Gaussian approximation being far beyond 90 percent through a wide range of $V$-parameter values. Solving (6) for lateral TE-polarization yields in terms of the normalized parameters [4]:

$$
w_{m}=\frac{w_{g}}{\sqrt{2} \pi}\left\{1+\frac{\sin u}{u}+\frac{1+\cos u}{v}\right\}
$$

in which $w_{g}$ is the lateral guide width. Fig. 3 shows a plot of the dependance of $w_{m} / w_{g}$ on the lateral $V$-parameter. Because lateral contrasts are usually small the formula is equally well suited to TM-polarization.

Using this assumption we find:

$$
\begin{aligned}
& U_{e}(y, z)= \exp \left[-y^{2} / w_{m}^{2}+\left(z-z_{c}\right) / l_{c}-j \beta z\right] \\
& z \leq z_{c} \\
&=0, \quad z>z_{c}
\end{aligned}
$$

in which $l_{c}$ is the prism-coupling length governing the $z$-dependence. Substitution of (4), (5), and (8) (with $\beta=$ $n_{p} k_{0} \cos \theta$ ) into the integrals of (1) yields:

$$
\begin{gathered}
\iint\left|U_{i}(y, z)\right|^{2} d y d z=\frac{\pi}{2} w_{y 0} w_{z} \\
\iint\left|U_{e}(y, z)\right|^{2} d y d z=\frac{\sqrt{ } \pi}{2 \sqrt{2}} w_{m} l_{c} \\
\iint U_{i}(y, z) U_{e}^{*}(y, z) d y d z \\
=\sqrt{ } \pi \int_{-\infty}^{z_{c}} Q(z)\left\{\frac{w_{y 0}}{w_{y}(z)}\right\}^{1 / 2} e^{-z^{2} / w_{z}^{2}} \\
\cdot \exp \left[\left(z-z_{c}\right) / l_{c}+\frac{1}{2} j \arctan z / b_{y 0}\right] d z
\end{gathered}
$$

in which

$$
Q(z)=w_{m} w_{y}(z) /\left[w_{m}^{2}+w_{y}^{2}(z)+j w_{m}^{2}(z) / b_{y 0}\right]^{1 / 2} .
$$

Substitution of (9), (10), and (11) into (1) leads to an expression for $\eta$ containing a single integration in $z$. Numerical evaluation of this expression as a function of $w_{y 0}$ with the effective mode width $w_{m}$ as parameter yields the results plotted in Fig. 4 with solid lines. For $l_{c}$ and $z_{c}$ we substituted the optimal values for the two-dimensional coupling case $\left(l_{c}=1.48 w_{z}\right.$ and $\left.z_{c}=0.73 w_{z}[4]\right)$.

One can draw some important conclusions based on Fig. 4. For very wide waveguides the maximum coupling efficiency approaches 80 percent as in the two-dimensional situation, the maximum occuring at $w_{y 0}=w_{m}$, i.e., the input beam and the mode have the same width. For smaller mode widths the maximum coupling efficiency is reduced and the position of the maximum is fixed approximately at $w_{y 0} \simeq 6 \mu \mathrm{m}$. 


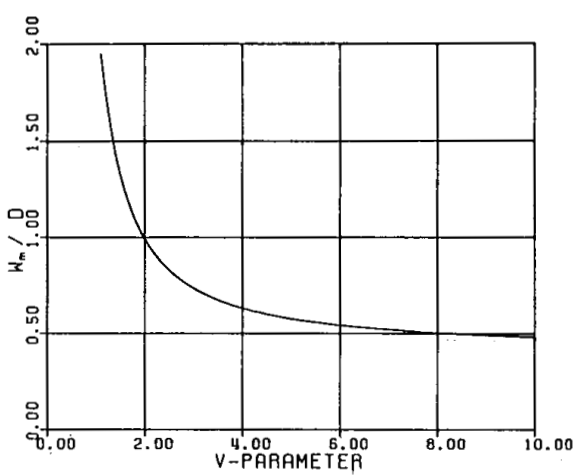

Fig. 3. The ratio $w_{m} / w_{g}$ as a function of the lateral $V$-parameter.

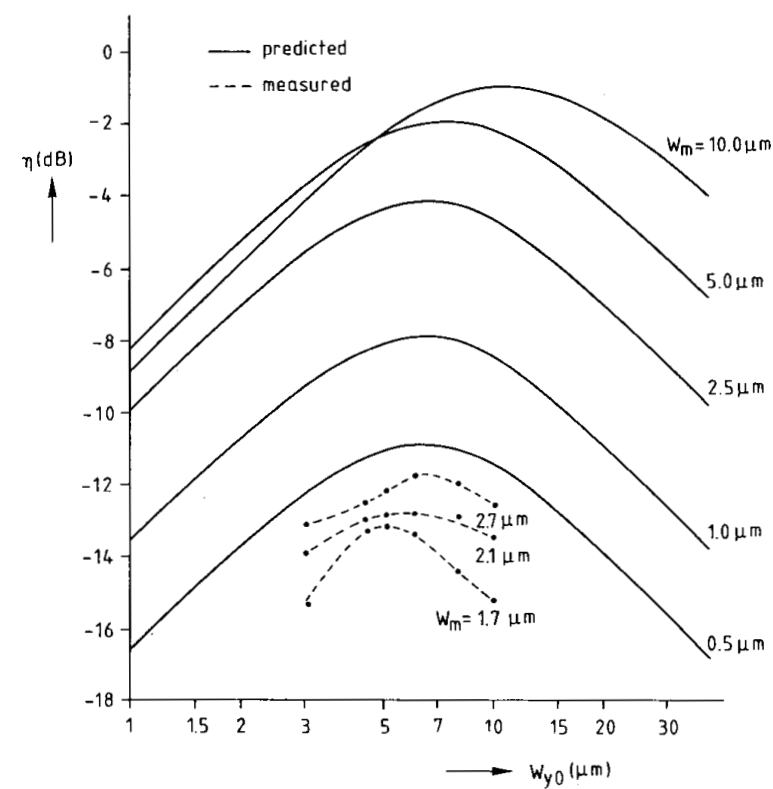

Fig. 4. The coupling efficiency as a function of the (lateral) focal width $w_{y 0}$ for different values of the effective mode width $w_{m}$.

As can be seen it makes no sense to apply focal (half) widths less than $6 \mu \mathrm{m}$ ( at $0.6328 \mu \mathrm{m}$ ) for narrow waveguides. Furthermore the efficiency loss in applying considerably wider beams $(20-30 \mu \mathrm{m})$ is relatively small.

Physically these results may be explained as follows. For small focal widths of the input beam it is impossible to match the width of the input beam to the mode width throughout the entire coupling region due to the beam widening (as a result of diffraction) before and behind the focal plane. Furthermore, the curvature of the wavefronts will destroy the phase-match along the optical axis. In applying a wider input beam the phase-front curvature in the coupling region is reduced and, clearly, this increases the coupling efficiency up to focal widths of $6 \mu \mathrm{m}$, independent of the waveguide width.

We checked the computed results experimentally for a number of focal widths and waveguide widths. The values found experimentally are also indicated in Fig. 4. The shape of the experimental curve and the position of the maximum compare well with the theoretical results. The absolute levels differ a few decibels; we did not invest much time in absolute calibration.

\section{B. Coupling Selectivity}

Focusing also affects the mode selectivity of the coupling. A small incidence angle deviation $\Delta \theta$ (in (4)) contributes a factor $\exp \left(+j n_{p} k_{0} z \Delta \theta \sin \theta\right)$ to the integrand of (11). Fig. 5 displays the angle dependance of $\eta$ for two different values of $w_{y 0} / w_{m}$ with $w_{m}=1.7 \mu \mathrm{m}$. For a wide input beam the width is approximately the same as for the transverse coupling configuration. This might be expected because of the plane character of the input beam. For small beamwidths the angular width of the coupling curve increases, but not dramatically.

These results were also verified experimentally on a 3.5- $\mu \mathrm{m}$-wide waveguide with $w_{m}=1.7 \mu \mathrm{m}$. Fig. 6(a) shows the measured coupling spectrum of the first three lateral modes of the waveguide excited with a wide beam $\left(w_{y_{0}} / w_{m} \simeq 12\right)$. The halfwidth of the $\mathrm{HE}_{00}$ curve at the $1 / e^{2}$ level is approximately 0.00027 , corresponding to $w_{\eta}$ $\simeq 0.02^{\circ}$ (inside the prism). Fig. 5 also predicts $0.02^{\circ}$. Fig. 6(b) shows the spectrum resulting from excitation with a strongly focused beam $\left(w_{y 0} / w_{m} \simeq 1.5\right)$. The broadening of the spectrum $\left(w_{\eta} \simeq 0.07^{\circ}\right)$ appears to be considerably greater than predicted by Fig. $5\left(w_{\eta} \simeq\right.$ $0.03^{\circ}$ ). A possible explanation of this phenomenon may be the following. Wang and Laybourn [7] have calculated and Miller et al. [5], Simova et al. [6], and the present authors experimentally determined from the diffraction pattern of the field coupled out of a waveguide that radiation does not occur along a plane at an angle $\theta$ with the bottom of the prism, but along a conical surface with the top in the coupling region. Based on reciprocity one therefore concludes that, in order to obtain good efficiency, the input beam should be focused along a conical surface. It is difficult to realize this in practice. If we do not fulfill this requirement, the field match in the coupling region will be violated leading most likely to the spectral broadening observed in Fig. 6(b). The direction of the broadening is in agreement with this explanation. The curvature of the conical surface of the output field is in the direction of smaller values of $\theta$, i.e., higher values of $N_{\text {eff. }}$ This means, conversely, that if we apply a noncurved input beam the broadening of the coupling spectrum will occur in the direction of higher $N_{\text {eff }}$ which has been noted.

\section{Other Mechanisms Causing Selectivity REDUCTION}

Refraction of a laterally focused input beam by the front face of the prism causes the incidence angle within the prism to become dependent on the skew angle. Measurement of the angular broadening of the coupling efficiency of a focussed beam to a waveguiding film (without lateral confinement) showed that this effect is much smaller than the broadening observed in Fig. 6(b). 


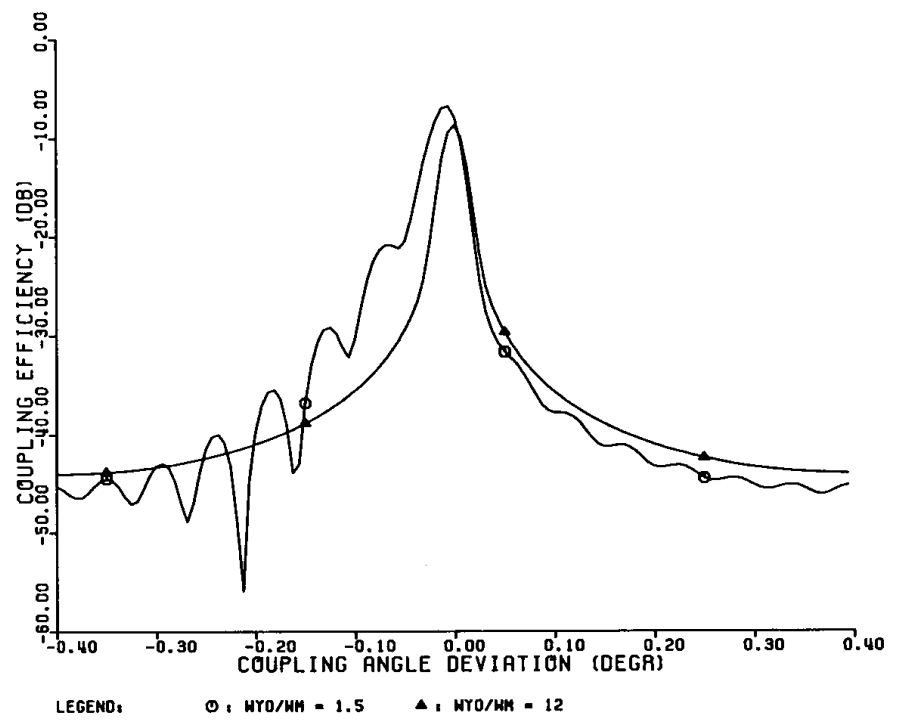

Fig. 5. The coupling efficiency as a function of $\Delta \theta$ for two different focal widths (with $w_{m}=1.7 \mu \mathrm{m}$ ).

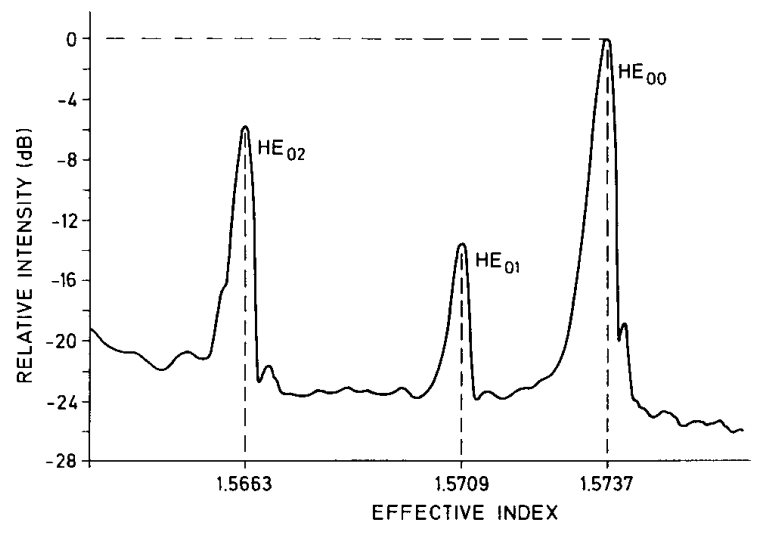

(a)

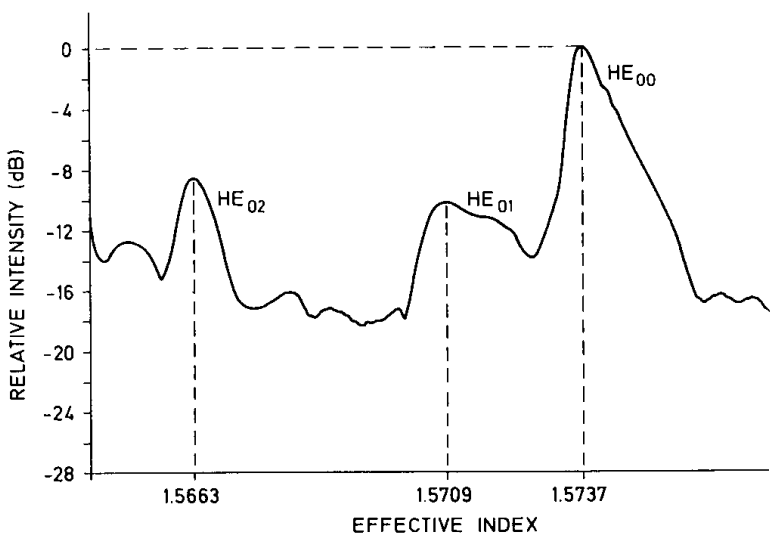

(b)

Fig. 6. Experimental record of the coupling efficiency for three lateral modes as a function of $\theta$ for two different focal widths ( $w_{m} \simeq 1.7 \mu \mathrm{m}$ for the fundamental mode): (a) $w_{y 0} / w_{m} \simeq 1.5$. (b) $w_{y 0} / w_{m} \simeq 12$.
If the angles of the front and the back faces of the input prism are poorly chosen multiple reflections may couple into the waveguide at unexpected angles. If the direct beam does not couple to a waveguide mode it is totally reflected by the bottom of the prism, but also by the chromium coated back face. Due to the high prism index the reflection coefficient at a glass-air interface is also high, so that multiple reflected beams decay slowly. The problem can be analyzed by tracing the possible ray paths, and can be avoided by choosing proper prism angles (for our experimental configuration: front face angle $60^{\circ}$; back face angle $75^{\circ}$ ) and by making the chromium coated backface diffuse.

We note the following concerning the use of polarizers to attenuate the input power, for example when a sensitive camera tube is employed. If a randomly polarized laser is applied (or a linearly polarized one at $45^{\circ}$ ) the complementary polarity will be much stronger than the intended one. Through the tails of the coupling curves this may lead to excitation of unwanted modes in the substrate. This problem may be solved by using neutral filters.

For very short coupling lengths the prism and the input beam have to be aligned with great care in order to avoid reduction of the coupling efficiency and broadening of the angular coupling curve. Improper alignment resulted in dramatic broadening (up to $4 x$ ) for a $0.4-\mathrm{mm}$ coupling length. For $1.5 \mathrm{~mm}$ the effects are much smaller.

A problem in the excitation of ridge-guide modes is that the coupling lengths for the ridge and the cladding region are different precluding optimum alignment for both the ridge and the cladding region. If the alignment is optimized for the guided mode the selectivity for the excitation of lateral cladding modes may be severely degraded. This may cause lateral cladding modes to be excited simultaneously with the guided modes. The problem is 
aggravated if the focal width of the input beam is chosen substantially greater than the waveguide width $w_{g}$ because then a large part of the flux hits the cladding region.

The above problem can be reduced by choosing $l_{c} \gg$ $w_{z}$ and by applying low contrasts. If other requirements preclude such a solution it is important to take good notice of the possible effects.

\section{Conclusions}

We have concluded that coupling of light into narrow optical waveguides is only possible if high coupling losses due to diffraction effects in the coupling region are accepted. If high coupling efficiencies are required a wide waveguide should be applied in the coupling region and tapered to the required width afterwards. If a maximal coupling efficiency is not required the application of an "oversized" input beam is advantageous. For narrow waveguides matching the beam width in the focal plane to the mode width reduces both selectivity and coupling efficiencies. For the experimental waveguide configuration described in this article focal widths of 20 to $30 \mu \mathrm{m}$ combine reasonable coupling efficiencies with good selectivity. We note that a large focal width reduces the sensitivity of the coupling to environmental vibrations. Furthermore the use of large coupling lengths is recommended, short coupling lengths reducing the selectivity (especially next to the ridge) and making the coupling more sensitive to input beam misalignment.

\section{ACKNOWLEDGMENT}

The authors wish to thank Prof. G. A. Acket for his helpful suggestions and $F$. van Ham for his assistance in the experimental part of the work.

\section{REFERENCES}

[1] P. K. Tien, R. Ulrich, and R. J. Martin. "Modes of propagating light waves in thin deposited semiconductor films," Appl. Phys. Lett., vol. 14, pp, 291-294, 1969

[2] P. K. Tien and R. Ulrich. "Theory of prism-film coupler and thinfilm light guides," J. Opt. Soc. Amer. . vol. 60, pp. 1325-1337. 1970

[3] R. Ulrich, "Theory of prism-film coupler and thin-film light guides." J. Opt. Soc. Amer., vol. 60, pp. 1337-1350, 1970.

[4] H. G. Unger, Planar Optical Waveguides and Fibers. Oxford: Clarendon. 1977, sect. 2.7

(5) C A. Millar, R H Hutchins, and P. J R Laybourn "Modes of a 3-dimensional sandwich-ribbon optical waveguide." Microwave Opt. Acoust., vol. 1, pp. 27-33, 1976

6] P. Simova, I. Savatinova, and L. Tsonev, "Propagation characteristics of Ag " ion-exchanged stripe waveguides," Appl. Phys., vol. 22. pp. 237-240, 1980
[7] D. K. Wang and P. J. R. Laybourn, "Coupling between prism and rectangular optical waveguide," Proc. Inst. Elec. Eng., vol. 130, pp. 255-260, 1983.

[8] M. K. Smit, C. J. van der Laan, and G. A. Acket, "Aluminium oxide films for integrated optics." Thin Solid Films, vol. 138, pp. 171-181, 1986.

[9] M. K. Smit. "Sputtered planar optical waveguides," in Sensors and Actuators. Proc. S \& A Simp. Twente Univ. Tech. (Enschede, the Netherlands), Oct. 30-31, 1986.

[10] H. Kogelnik, "Imaging of optical modes, "Bell. Syst. Tech. J., vol. 44 , pp. $455-494,1965$

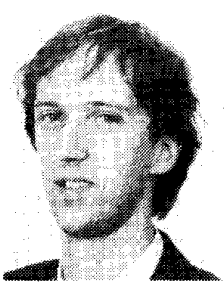

Wim A. Pasmooij graduated in electrical engineering from the Delft University of Technology in 1986. During his study he was engaged in in tegrated optics research

He is currently employed at the Dr. Neher Laboratory of the Netherlands PTT, where he is in volved in research on Electromagnetic Compatibility (EMC).

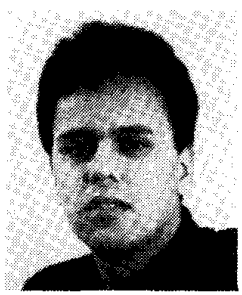

Peter A. Mandersloot was born in Moordrecht The Netherlands, on February 9, 1959. He graduated in electrical engineering from the Delft University of Technology in 1986. During his study he was engaged in research on integrated optics.

In 1987 he joined the Netherlands PTT, where he is involved in both the introduction and standardization of newly developed transmission de vices.

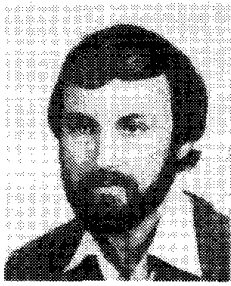

Meint K. Smit was born in Vlissingen, the Neth erlands. on January 5,1951 . He graduated in electrical engineering from the Delft University of Technology. in 1974

From 1974 to 1981 he was engaged in research on radar remote sensing, first with the Netherlands Interdepartmental Working community for the Application of Remote Sensing Techniques (NIWARS), and since 1976 with the Delft University of Technology. Since $198 \mathrm{I}$ he has worked in the field of optical communication with empha-

sis on integrated optics.

Mr. Smit is a member of the Netherlands Society for Electronic and Radio Engineering (NERG). 